\title{
Research about key technologies of simulation training systems based on Variable Message Format (VMF)
}

\author{
Fei Gao, Xue-jun Bi, Xiao-zhen Wang, Juan Liu \\ Academy of Armored Force Engineering, Beijing 100072, China.
}

Keywords: List the, keywords covered, in your paper.

\begin{abstract}
With the development of terminal equipment system based on information technology, simulation training becomes popular in the military field because of its convenience, efficiency, scalability, etc. However, it is hard for both the existing simulation training system simulation module and the actual equipment module to match properly. It is for people to operate one vie the other. This paper is about how to build the high level architecture (HLA) of simulation system and design data exchange gateway vie VMF, data packet transmission standard, to make simulation training close to the real equipment and improve the effectiveness of training.
\end{abstract}

\section{Introduction}

As information industry goes deeper, information terminal equipment is assigned to various professional fields. It provides the material basis for the formation of combat information system. In the military field, the medical field and teamwork training field, simulation training, regarded as the information system, is an important form of training to support simulation system. It is an important method for the construction of simulation training and an essential way to take advantage of the simulation training, when people use mounting system to make trainees to complete training under the same or similar mounting environment.

When it comes to the actual situation in the system, the main reasons that a large number of simulation systems cannot interconnect with actual equipment are listed below:

(1)Large numbers of simulation system have defects in the design concept. There is not a good interface constructed to support simulation training system, and it cannot be well connected with the actual equipment;

(2)Through the transformation of the previous simulation system, it facilitate the original system tightly coupled with a dedicated interface module to achieve the connection mounting system;

(3) Reasons listed above cause a lot of resources waste, which brings tremendous amount of work for developing the simulation training system and goes against to the reuse of existing software resources.

\section{Using VMF as a way of data transmission}

\section{VMF concept}

Under the condition of digital information transmission should consider the actual equipment. Increasingly demanding complex electromagnetic environment, no fixed infrastructure, mainly rely on the case of Ad Hoc network, VMF is an ideal way to transmit reliable data. It was proposed by the US military to standardize message format for ground forces command and control system, in a message on the adaptability and effectiveness of excellent performance.

\section{VMF advantage}

The four advantage are listed below:

(1)Information coding standard of variable message format is bit-oriented. Messages are passed vie binary code. It can not only reduce the amount of data transmitted, but also improve machine automatically recognize and deal with the message easily;

(2) Because of its coding standards, VMF can make use of network resource to enhance the adaptability and effectiveness of the message; 
(3)According to the literal meaning of VMF, the specific format and length of VMF can be adjusted in practical application;

(4)Because of its flexible syntax, VMF only allow users to send necessary information. The size of the necessary information could be changed, thereby reducing the amount of data transmitted and saving bandwidths.

\section{VMF Format}

Each packet of VMF message standards is identified by the form of Kn.m. K stands for the packet belongs to the series, $\mathrm{n}$ is the packet domain number; and $\mathrm{m}$ is the message number. As is written below, each format reports text description of the mandatory fields and optional fields in different combinations to compose a message to meet the needs of all kinds. Optional fields based on the value of the indicator field to determine whether, VMF contains fields appear Indicator (FPI), field duplicate indicator (FRI), grouping appear indicator (GPI) and group instruction is repeated four indicators (GRI) device. DFI / DUI (Data Domain Identifier / data using the identifier) is a set of numbers used to identify the data elements can be used as a reference for the data element dictionary, as shown below.

Table 1 VMF Format

\begin{tabular}{|c|c|c|c|c|c|c|c|}
\hline Kn.m & & & & & & & \\
\hline Message $\mathrm{F}$ & & & & & & & \\
\hline Message $\mathrm{F}$ & tion & & & & & & \\
\hline $\begin{array}{c}\text { Index } \\
\text { Number }\end{array}$ & DFI/DUI & $\begin{array}{c}\text { DUI } \\
\text { Name }\end{array}$ & Bits & Type & $\begin{array}{c}\text { Group } \\
\text { Code }\end{array}$ & Repeat Code & Explain \\
\hline
\end{tabular}

\section{The use of HLA simulation system architecture}

HLA, distributed by the Defense Modeling and Simulation Office (DMSO), proposed a new simulation standard. Because of its full-functioning feature and high reliability, it gradually becomes a hot topic in the field of military simulation. As a common technical framework, it defines the entire simulation process principles and interface standards. Its main components are: each individual simulation applications-Federate, the basic elements of the real world mapping federate internally-the object under the guidance of a specific task, member states can interact, support software and related components grouped together-Federal; HLA machines running on each node, providing a platform software-RTI federate between system management (see Figure 1 ).

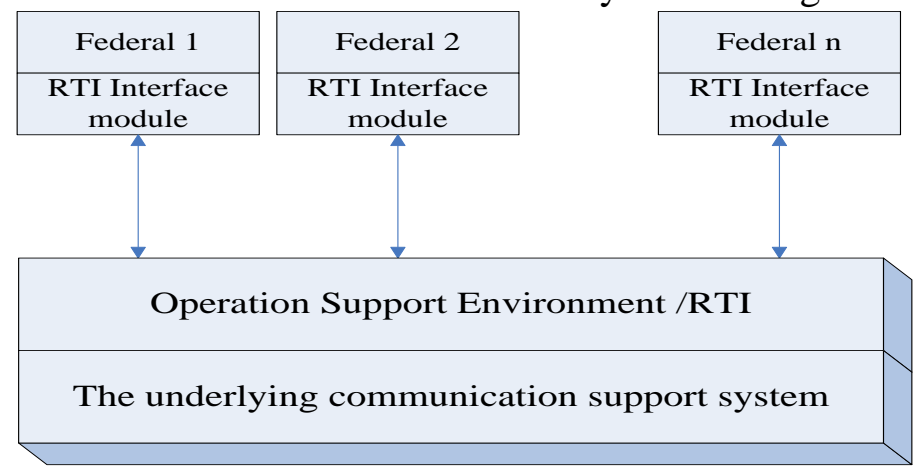

Fig. 1 HLA Running Framework

HLA system as the basis for interoperable and reusable, object model specification template HLA OMT is an important part, OMT using seven categories form a hierarchical class methods to describe and define the simulation system object classes and interactive classes, including: Identify the table object model, the object class structure table, interactive class structure table, the data attribute table, parameter table, the attribute table and parameter table type auxiliary table, routing table space. 


\section{VMF-HLA The idea of data exchange gateway}

Essentially, VMF-HLA Gateway is a member of HLA federate. That is why the process of establishing a gateway VMF-HLA is very similar to the common development process of HLA federate. In practical application, VMF message structure has a greater stability, the message structure, processing rules and semantics, generally not larger changes, but the simulation system does have greater randomness, and for this reason, and there is not a panacea VMF-HLA gateway can interconnect any simulation system with real equipment system, which must be for a specific simulation system, which adapts to establish gateways to handle the corresponding data exchange tasks.

Mounting between the environment in the form of command information systems VMF tactical message exchange is through a unified message transmission service implemented to solve the interconnection and mounting environment, interoperability, VMF-HLA Gateway requires the use of exactly the same environment with real equipment the transmission interface, while VMF-HLA HLA federates and as a gateway to achieve access via RTI bus and simulation systems, so as to VMF-HLA gateway supports mounting command Information System and semi-physical simulation system interconnect simulation training system the basic structure ( Figure 2), which joined VMF-HLA HLA federation gateway as a federal member responsible for mounting between the environment and simulation system for data exchange. The VMF-HLA gateway internal constituted by various functional components (as shown in Figure 2).

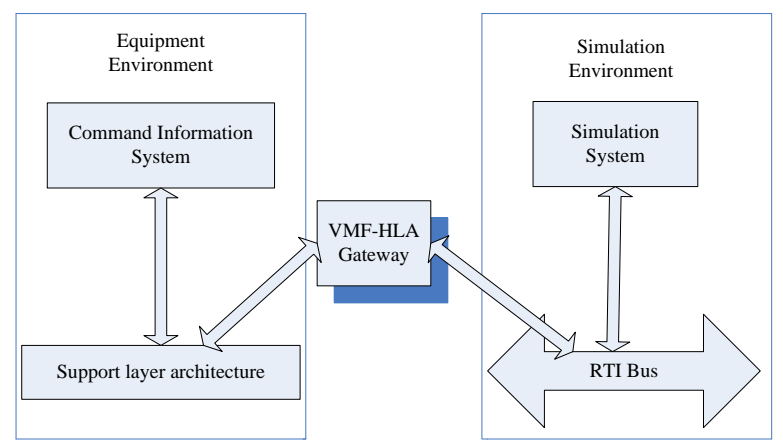

Fig . 2 The basic framework of WMF-HLA gateway support training system

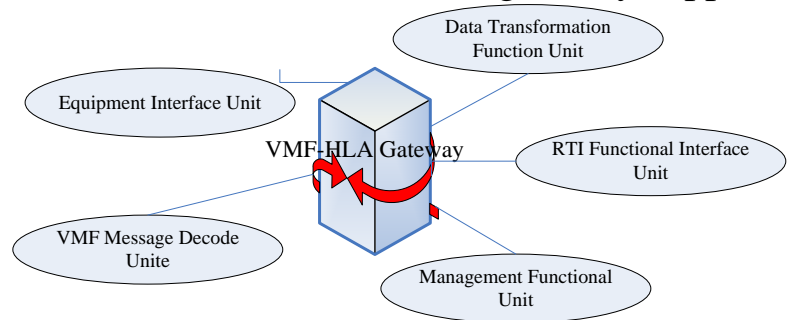

Fig. 3 The composition of the VMF-HLA gateway

Among them, the real equipment has access to function unit for data communication via the existing general support layer member and command information system, RTI interface functional units interact through RTI bus and simulation systems. VMF codec packets are generated by the VMF message functional unit standard describes software tools codec for handling messages tactics, tactical message data conversion unit responsible will receive between VMF form and simulation systems in the form of federation object model conversion. Time management coordinates RTI bus and mounting time combat environment advance through the HLA time management mechanisms and integration system components.

VMF-HLA gateway is established under the help of specific software tools (see Figure 4 ) : 


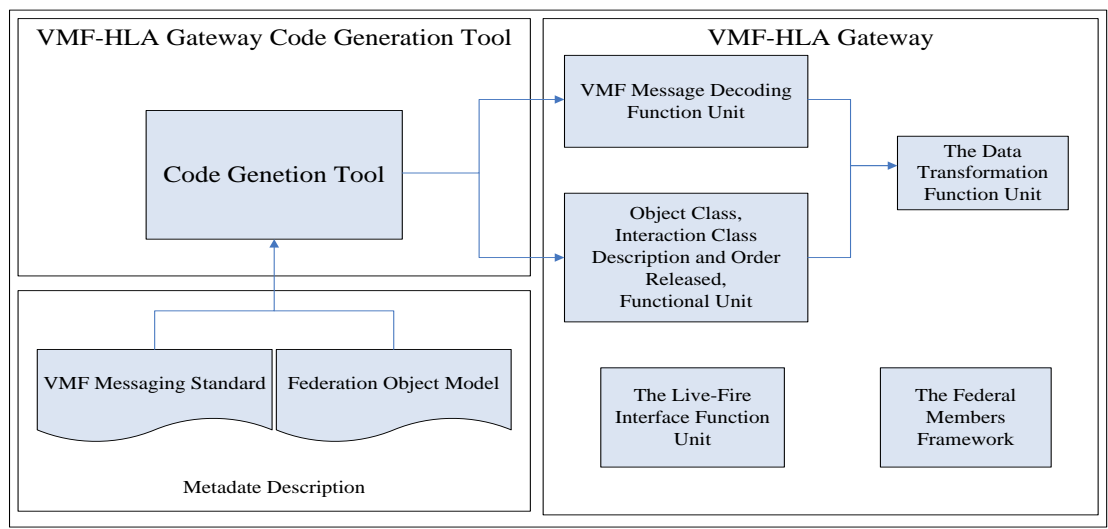

Fig. 4 Live-fire access module internal structure and process

\section{VMF-HLA Data exchange gateway design scheme}

VMF-HLA gateway internal structure:

(A) VMF-HLA gateway internal structure :

VMF-HLA gateway is made up of mounting internal access module (Figure 3).

(B) With integrated access module mounting member supporting layer, and command information systems for communication, its internal structure and processes (Figure 5).

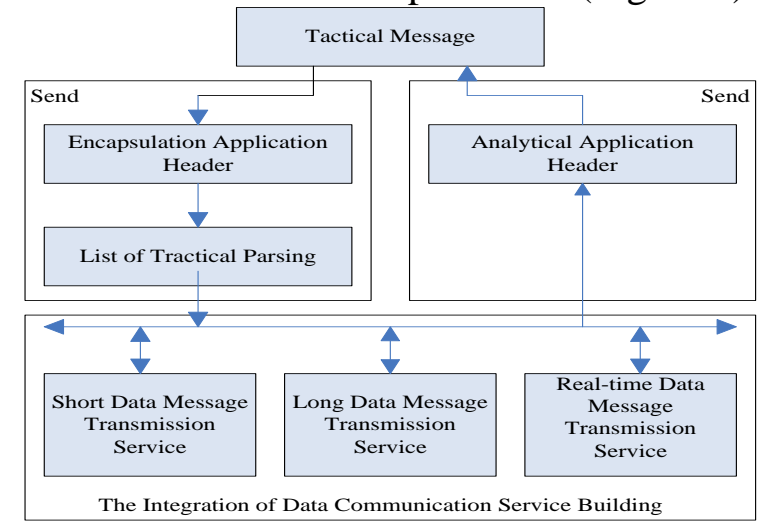

Fig. 5 Live-fire access module internal structure and process

(C) VMF message codec module code is automatically created by the aid of software tools based on tactical messaging standards, including $\mathrm{C}++$ data structures used to describe the structure of the message, as well as improve the efficiency, taking hard-coded for each type of message according to the structure and its message processing rules to generate the corresponding $\mathrm{C}++$ code codec.

(D)The data conversion module is produced under the aid of software tools. By parsing the federal document object mode, each object class, interaction class and ordering process produce a corresponding announced $\mathrm{C}++$ code, implement object class, interactive class data and form of tactical messages between conversion.

(E) RTI interface module is part of the federal member framework, RTI interface module handles added to secede during the execution of the necessary work, and is responsible for ordering and publish the required object class, interactive class, its process.

(F) Time Management module is responsible for obtaining the current simulation time from RTI bus, according to the training mission in war time setting parameters into the current war time, and this time release to the environment through real-time system installation service member training systems.

(G) VMF-HLA federates deployed as a gateway between the mounting system and simulation system, enabling tactical message HLA object classes, interactive conversion between classes , the process ( Figure 8). 


\section{Summary}

According to the current situation of simulation training, this paper provides two pieces of innovative ideas:

(1) People could use gateway technology to build simulation training system, which does not change too much on the original simulation system but achieve the purpose of interoperability and interoperability in large-scale modification and mounting environment;

(2) Because code generation technology can automatically generate most of the gateway program code, it reduces the workload and the difficulty of the process of building simulation training system gateway development.

To Summarize, it promotes the construction of the simulation training system, and fro the long run it is meaningful to the military field.

\section{References}

[1] Edward P. Dunn, III, George Rumford. TENA: A Domain-Specific Architecture for Live Participants[ED/OL],00s-siw-107,http://www.sisostds.org/SIW/00spring/SIW_TE.htm,Date/Ti me:05/03/02.

[2] M. Kim and J. Han, "CHRONICLE: a two-stage density-based clustering algorithm for dynamic networks,” in Proc.12th Int. Conf. on Discovery Science, Porto, Portugal, 2009, pp. 152-167.

[3] TAO: A High performance, Real-time Object Request Broker (ORB)[EB/OL]. http://www.cs.wustl.edu/ schmidt/TAO.html, Last modified 26 May 2004

[4] VMF TIDP-TE-2000,Variable Message Format(VMF) Technical Interface Design Plan (Test Edition)[S].

[5] High level architecture run-time infrastructure programmer's guide 1.3 version 4[OL]. http://www.dmso.mil

[6] Rumford, George J Minh Vuong. Foundation initiative 2010: the design of the second TENA middleware Prototype [EB/OL]. http://www.sisostds.org/siw, 2003

[7] L. Backstrom, D. Huttenlocher, J. Kleinberg, and X. Lan, "Group formation in large social networks: membership, growth, and evolution,” in Proc. 12th ACM SIGKDD int. Conf. on Knowledge Discovery and Data Mining, Philadelphia, USA, 2006. pp. 44-54.

[8] L. Tang, H. Liu, J. Zhang, and Z. Nazeri, "Community evolution in dynamic multi-mode networks," in Proc. 14th ACM SIGKDD Int.Conf. on Knowledge Discovery and Data Mining, Las Vegas, Nevada, USA, 2008, pp. 677-685.

[9] X. Xu, N. Yuruk, Z. Feng, and T. A. Schweiger, "SCAN: a structural clustering algorithm for networks," in Proc. 13th ACM SIGKDD Int..Conf. on Knowledge Discovery and Data Mining, San Jose, California, USA, 2007, pp. 824-833.

[10] S. Lin and H. Chalupsky, "Discovering and explaining abnormal nodes in semantic graphs," IEEE Trans. Knowl. Data Eng., vol. 20,no. 8, pp. 1039-1052, Aug. 2008. 